\title{
GESTIÓN DEL RIESGO CREDITICIO Y SU INFLUENCIA EN EL NIVEL DE MOROSIDAD DE LA FINANCIERA CREDINKA S.A. - AGENCIA QUILLABAMBA - PERÍODO 2015
}

\section{Credit risk management and its influence on the level of delinquency of the financial company Credinka S.A. - Agencia Quillabamba - Period 2015}

\author{
Yeni Araceli Yépez cajigas ${ }^{1, \text { : }}$ \\ ${ }^{1}$ Universidad Andina del Cusco, Cusco, Perú \\ ”yyepez@uandina.edu.pe
}

\begin{abstract}
Resumen
Las entidades financieras enfrentan riesgos externos relacionados principalmente a la coyuntura económica del país en el cual están establecidas (riesgo país, cambiario, de mercado y legal) y riesgos internos relacionados a la incertidumbre inherente a las operaciones que realiza (riesgo crediticio, liquidez y operacional), los cuales pueden afectar el resultado de la entidad. La gestión del riesgo crediticio es el tipo de riesgo más importante al que debe hacer frente cualquier institución financiera, un indicador de este riesgo es el nivel de morosidad; es decir, la proporción de su cartera que se encuentra en calidad de incumplimiento. La presente investigación se llevó a cabo con el propósito de determinar cómo influye la gestión del riesgo crediticio en los niveles de morosidad en la Financiera CREDINKA S.A. - Agencia Quillabamba - periodo 2015. El tipo de investigación es descriptivo y correlacional. El método de investigación aplicado es el método hipotético deductivo - inductivo. El diseño es no experimental es de corte transeccional o transversal. La población de estudio está conformada por 4,079 clientes, siendo la muestra de 124 y los 22 trabajadores de la entidad financiera. Como el resultado del análisis de la información se ha confirmado lo siguiente: Una adecuada gestión del riesgo de crédito en base al desarrollo de estrategias, políticas y herramientas de aprobación, seguimiento y recuperación de los clientes; influye disminuyendo niveles de morosidad de la institución financiera CREDINKA - Agencia Quillabamba.
\end{abstract}

Palabras clave: Riesgo; Riesgo Crediticio; nivel de Morosidad.

\begin{abstract}
Financial institutions face external risks related mainly to the economic situation of the country in which they are established (country, currency, market and legal risk) and internal risk related to the uncertainty inherent to the operations carried out (credit risk, liquidity and operational), which can affect the result of the entity. Credit risk management is the most important risk that any financial institution must cope, an indicator of this risk is the level of non-performing loans; i.e., the proportion of your portfolio that is in non-compliance with quality. This research was conducted in order to determine how influences the management of credit risk at the levels of non-performing loans in the financial CREDINKA S.A. - Agency Quillabamba - 2015 period. The type of research is descriptive and correlational. The research method applied is the hypothetical deductive method - inductive. The design is non-experimental is cutting transactional or transverse. The study population is formed by 4,079 customers, being the sample of 124 and 22 workers of the financial institution. As the results of the analysis of information has confirmed the following: proper management of credit risk based on the development of strategies, policies and tools for approval, monitoring and recovery of clients; decreasing levels of non-performing loans of the financial institution CREDINKA - agency Quillabamba influences.monitoring and recovery of clients; decreasing levels of non-performing loans of the financial institution CREDINKA - agency Quillabamba influences.

Keywords: risk; Credit risk; level of non-performing loans.
\end{abstract}

Citar como: Yépez, Y. (2018). Gestión del riesgo crediticio y su influencia en el nivel de morosidad de la financiera Credinka S.A. - Agencia Quillabamba - Período 2015. Rev Yachay, 7(1) ,417-423.

Recibido: 16-09-2018; Aceptado 07-12-2018

Rev. Yachay volumen (7) Número (1), enero-diciembre 2018 


\section{Introducción}

En el Perú, la economía ha venido experimentado un crecimiento económico sostenido y elevado, factor clave que ha contribuido tanto a la expansión de las colocaciones del sistema financiero como al aumento significativo del riesgo crediticio que enfrentan las instituciones financieras. La financiera CREDINKA - Agencia Quillabamba, no es ajena a los cambios que se presentan en el mercado microfinanciero, donde la competencia por aumentar la cartera de clientes y préstamos influye en el incremento del riesgo crediticio debido a que utilizan estrategias como: bajas tasas de interés, mayores plazos, montos, mínima documentación, no exigir garantías, etc. todo esto motiva a que el cliente pueda tener un abanico de instituciones financieras para escoger, desde un banco hasta usureros (prestamistas informales). La presente investigación también tiene como objetivos específicos: a) Describir las características generales de la gestión riesgo crédito, en todas las fases del proceso de crédito: estrategias de gestión del riesgo crediticio, políticas de crédito y herramientas de evaluación de crédito en la Financiera CREDINKA - Agencia Quillabamba. b) Analizar y evaluar los niveles de morosidad de la entidad en estudio, en la cartera atrasada o morosa, la cartera de alto riesgo y la cartera pesada en la Financiera CREDINKA - Agencia Quillabamba y c) Proponer acciones de gestión del riesgo crédito, con miras a disminuir los niveles de la morosidad en la Financiera CREDINKA - Agencia Quillabamba. Los resultados de la investigación son relevantes debido a que se conocerá la calidad de la gestión de riesgos crediticios, así como los niveles de morosidad en la Financiera CREDINKA - Agencia Quillabamba, sobre la base de esto se podrán sugerir acciones tendientes a mejorar ciertas deficiencias en estos dos aspectos sumamente importantes en la gestión de las instituciones financieras. El presente trabajo de investigación, es de mucha importancia por qué tiene el propósito de analizar la situación actual en la cual se encuentra el ente de estudio en cuanto a la gestión de riesgos crediticios y morosidad, ya que el presente trabajo cuenta con encuestas realizadas tanto a los trabajadores como a los clientes de la Financiera CREDINKA - Agencia Quillabamba.

\section{Materiales y Métodos}

El estudio corresponde al tipo de investigación aplicada en razón que los resultados son aplicados para solucionar el problema de la deficiencia de la gestión del riesgo crediticio y su influencia en los niveles de la morosidad en la Financiera CREDINKA S.A. - Agencia Quillabamba. Así mismo, se define el tipo de investigación según la naturaleza de los instrumentos aplicados es de tipo cuantitativa. Finalmente, la ocurrencia de los hechos es prospectiva. Es descriptivo, ya que se pretende conocer las características en detalle de cada una de las variables estudiadas. Es correlacional, puesto que se pretende establecer la relación entre las variables de estudio; por tanto, en vista que no se van a manipular ninguna de las variables es no experimental; por lo que, su aplicación se desarrolla deliberadamente las variables, y se basa en hechos que ocurrieron; del mismo modo, se da en la realidad actual, con un enfoque retrospectivo y de actualidad. Las técnicas utilizadas en el presente trabajo de investigación fueron como sigue: Observación directa; encuestas; análisis de documentos; Internet; capturar la mayor información oportuna, precisa y confiable. Los instrumentos empleados en este trabajo de investigación fueron: Ficha de observación directa; cuestionario de encuesta; Ficha de recopilación de datos; las pruebas de estadísticas que se utilizó en el proceso de trabajo: Estadística descriptiva y la estadística inferencial; usualmente primero se utilizaron las descripciones y luego las inferenciales. Cuadros y gráficos estadísticos para variables cualitativos; se analizó en forma exclusiva las variables. Esto permitirá conocer y entender la forma como se vienen comportando los datos en cada variable.

\section{Resultados}

El análisis de datos se efectuó aplicando la estadística descriptiva - comparativa, tomando como base las frecuencias y los porcentajes de los analistas y jefes de negocio. Los mismos, se estructuraron en tablas y gráficos que permitió el análisis y la discusión contrastada con los fundamentos teóricos en los que se basa la investigación para demostrar las tendencias de las opiniones de los encuestados y darle, estadísticamente consistencia a la propuesta que conforma los objetivos del estudio. 


\section{Tabla 1}

Distribución de la muestra de estudio según características generales de los trabajadores de la entidad financiera.

\begin{tabular}{cccc}
\hline & DATOS GENERALES & Frecuencia & Porcentaje \\
\hline \multirow{2}{*}{ Sexo } & Masculino & 10 & $45,45 \%$ \\
& Femenino & 12 & $54,55 \%$ \\
Edad & TOTAL & $\mathbf{2 2}$ & $\mathbf{1 0 0 , 0 0 \%}$ \\
& Menos de 25 años & 7 & $31,82 \%$ \\
& de 26 a 35 años & 9 & $40,91 \%$ \\
Tiempo de servicio en la institución & de 36 a 45 años & 5 & $22,73 \%$ \\
& Más de 46 años & 1 & $4,55 \%$ \\
& TOTAL & $\mathbf{2 2}$ & $\mathbf{1 0 0 , 0 0 \%}$ \\
& Menos de 5 años & 15 & $68,18 \%$ \\
Formación académica & de 5 a 10 años & 6 & $27,27 \%$ \\
& de 11 a 20 años & 1 & $4,55 \%$ \\
& TOTAL & $\mathbf{2 2}$ & $\mathbf{1 0 0 , 0 0 \%}$ \\
& Título profesional universitario & 2 & $9,00 \%$ \\
& Título profesional de otras especialidades & 20 & $91,00 \%$ \\
\cline { 2 - 5 } & TOTAL & $\mathbf{2 2}$ & $100,00 \%$
\end{tabular}

En la Tabla 11, se aprecia que la mayoría de los trabajadores encuestados son de sexo femenino representan un 54,55\%. Del mismo, se observa que la muestra de estudio está formada en su mayoría por trabajadores cuya edad es 26 a 35 años. Así mismo, el 68,18\% de los trabajadores encuestados tienen el tiempo de servicio en la institución menos de 5 años. Finalmente, un buen porcentaje de los analistas y/o trabajadores revelaron que no tienen título profesional universitario, que representa el $91,00 \%$ de los encuestados.

Tabla 2

Distribución de la muestra de estudio según características generales de los clientes.

\begin{tabular}{cccc}
\hline \multirow{2}{*}{ DATOS GENERALES } & Frecuencia & Porcentaje \\
\hline \multirow{2}{*}{ Sexo } & Masculino & 45 & $36,29 \%$ \\
\cline { 2 - 4 } & Femenino & 79 & $63,71 \%$ \\
\cline { 2 - 4 } & TOTAL & $\mathbf{1 2 4}$ & $\mathbf{1 0 0 , 0 0 \%}$ \\
\hline Edad del cliente & Menos de 25 años & 19 & $15,32 \%$ \\
\cline { 2 - 4 } & de 26 a 35 años & 52 & $41,94 \%$ \\
\cline { 2 - 4 } & de 36 a 45 años & 41 & $33,06 \%$ \\
\cline { 2 - 4 } & Más de 46 años & 12 & $9,68 \%$ \\
\cline { 2 - 4 } & TOTAL & $\mathbf{1 2 4}$ & $\mathbf{1 0 0 , 0 0 \%}$ \\
\hline Tipo de crédito obtuvo & Crédito mes & 73 & $58,87 \%$ \\
\hline
\end{tabular}




\begin{tabular}{cccc}
\hline & \multicolumn{1}{c}{ Crédito de consumo } & 31 & $25,00 \%$ \\
\cline { 2 - 4 } & Crédito hipotecario & 7 & $5,65 \%$ \\
\cline { 2 - 4 } Tiempo obtuvo el crédito & Crédito agrícola & 13 & $10,48 \%$ \\
\cline { 2 - 4 } & TOTAL & $\mathbf{1 2 4}$ & $\mathbf{1 0 0 , 0 0 \%}$ \\
\cline { 2 - 4 } & Menos de 6 meses & 19 & $15,32 \%$ \\
\cline { 2 - 4 } & de 7 a 12 meses & 50 & $40,32 \%$ \\
\cline { 2 - 4 } & de 13 a 24 mes & 30 & $24,19 \%$ \\
\cline { 2 - 4 } & de 25 meses a más & 25 & $20,16 \%$ \\
\hline
\end{tabular}

En la

Tabla 22, se aprecia que la mayoría de los clientes encuestados son de sexo femenino representando un $63,71 \%$. Del mismo, se observa que la muestra de estudio está formada en su mayoría por clientes y/o prestamistas cuya edad esta entre 26 a 35 años. Así mismo, el 58,87\% de los clientes y/o prestamistas encuestados obtuvieron el crédito mes en la institución financiera. Finalmente, un buen porcentaje de los clientes expresaron que tienen obtuvieron el crédito de 7 a 12 meses, que representa el 40,32\% de los encuestados.

Tabla 3.

Gestión de riesgo crediticio realizada en

la institución financiera CREDINKA

\begin{tabular}{lcr}
\hline Detalle & Frecuencia & Porcentaje \\
\hline Buena & 5 & $22,73 \%$ \\
Regular & 15 & $68,18 \%$ \\
Malo & 2 & $9,09 \%$ \\
TOTAL & 22 & $100 \%$
\end{tabular}

En la Tabla 13, como se observa la gestión de riesgo crediticio en la institución financiera CREDINKA - Agencia Quillabamba - La Convención: el 68,18\% de los trabajadores encuestados manifestaron que es regular.

Tabla 3

Puntuación promedio del indicador gestión del riesgo de crédito

\begin{tabular}{ccc}
\hline Calificación & Operación & Promedio \\
\hline Muy Deficiente & $(27,50+19,50) / 2$ & 23,50 \\
Deficiente & $(31,80+19,10) / 2$ & 25,50 \\
Regular & $(12,45+15,00) / 2$ & 13,70 \\
Buena & $(1,25+3,00) / 2$ & 2,10 \\
Muy buena & 0 & 0,00 \\
TOTAL & & 65 \\
\hline
\end{tabular}


Tabla 4

Escala para calificar la gestión del riesgo de crédito

\begin{tabular}{cc}
\hline Calificación & Intervalos \\
\hline Muy Deficiente & 80 a 100 puntos \\
Deficiente & 60 a 80 puntos \\
Regular & 40 a 60 puntos \\
Buena & 20 a 40 puntos \\
Muy buena & 0 a 20 puntos \\
\hline
\end{tabular}

En cuanto al puntaje de calificación de la variable de gestión del Riesgo de Crédito es de 65 puntos (

Tabla 4), que equivale al nivel DEFICIENTE; de igual manera la tasa de Morosidad de la entidad en estudio es de $11.79 \%$ en el periodo 2015 que se incrementó con respecto al periodo anterior, que equivalente a un nivel DEFICIENTE. Los resultados se debieron también a la recesión económica que se vivió en la Provincia de la Convención como consecuencia del recorte presupuestal por regalías del canon y sobre canon gasífero.

\section{Discusión}

Con la información obtenida podemos afirmar que el objetivo general del presente trabajo de investigación que es la de: "Determinar cómo la gestión del riesgo crediticio influye en los niveles de la morosidad en la Financiera CREDINKA - Agencia Quillabamba - periodo 2015", se ha cumplido, siendo que la agencia hace una buena gestión en la Administración del Riesgo del Crédito aplicando estrategias, políticas y herramientas de evaluación a los créditos otorgados y según los resultados los índices de morosidad se debe principalmente a factores externos a la entidad financiera. Del análisis antes planteado, se encontró que existen imperfecciones en el mercado financiero del Perú - Cusco y estos se reflejan en la operatividad bancaria y más aún en la institución financiera, a la hora de determinar el riesgo crediticio y más específicamente, la determinación de las provisiones de insolvencia. Principalmente porque la cobertura de provisiones no es óptima. Para mejorar esta situación, se plantea soluciones estratégicas como las reservas anticíclicas (provisiones). Las mismas que aumentan durante la fase expansionista y, durante las épocas de recesiones, las provisiones específicas tenderían a aumentar. Es decir, su función es la de acumular recursos patrimoniales durante la expansión del producto y del crédito, los cuales habrán de emplearse o desacumularse durante la contracción del producto y del crédito, cuando el incremento de créditos malos reduzca la reserva estadística a través de las provisiones para proteger los créditos deteriorados durante el receso. Este procedimiento podría emplearse bajo los parámetros por el Banco Central y por el acuerdo de Basilea II, y por lo tanto promoverá la consecuencia de una mejor calificación de riesgo.

\section{Conclusiones}

La gestión del riesgo crediticio influye directamente en la disminución de los niveles de la morosidad en la Financiera CREDINKA Agencia Quillabamba - periodo 2015. Como el resultado del análisis de la información se ha confirmado lo siguiente: Una adecuada gestión del riesgo de crédito en base al desarrollo de estrategias, políticas y herramientas de aprobación, seguimiento y recuperación de los clientes; influye disminuyendo niveles de morosidad de la institución financiera CREDINKA - Agencia Quillabamba. Los niveles de morosidad de la entidad en estudio Financiera CREDINKA - Agencia Quillabamba, es de alta morosidad; porque alcanzo una tasa de $11,79 \%$ en el periodo 2015 , muy por encima de la morosidad promedio de la competencia que es de 4,40\% y también por encima de la tasa referencial mínima de 5\% señalado por Superintendencia de Banca y Seguro del Perú (SBS).

La implementación de las acciones de gestión del riesgo crédito, permitirá disminuir los niveles de la morosidad en la Financiera CREDINKA - Agencia Quillabamba. Deben ser basados con los objetivos de la institución financiera, influyendo positivamente en el nivel de morosidad de los clientes, lo cual significa una adecuada valoración del personal y/o analistas de créditos para una participación proactiva en la institución financiera. 


\section{Referencias Bibliográficas}

Aguilar Andía, G., \& Camargo Cárdenas, G. (2003). Análisis de la morosidad de las instituciones microfinancieras (IMF) en el Perú (Informe Técnico Final). Lima - Perú. Recuperado a partir de http://docplayer.es/3101897-Analisis-de-la-morosidad-de-lasinstituciones-microfinancieras-imf-en-el-peru-informe-tecnico-final.html

Añez, M. (2007). Aspectos básicos sobre análisis financiero de solicitudes de crédito industrial: casos prácticos para adiestramiento. Comisión de Valores Corporación Financiera Nacional del Ecuador.

Cisneros Salas, D. (2006). Gestión integral de riesgo (Superintendencia de Banca, Seguros - Superintendente). Lima - Perú. Recuperado a partir de http://logician.us/cracker/1429723950_38eacd88ee/gestion_integral_de_riesgos-dcisneros.pdf.

Díaz Quevedo, O. A. (2009). Determinantes del ratio de morosidad en el sistema financiero boliviano (Tesis). Bolivia: Banco Central de Bolivia.

Recuperado

a

partir

de

https://www.bcb.gob.bo/webdocs/publicacionesbcb/2015/11/51/3.\%20Determinantes\%20del\%20ratio\%20de\%20morosidad\%20e n\%20el\%20sistema\%20financiero\%20boliviano.pdf.

Guzmán Delgado, M. C. (2014). La administración del riesgo de crédito en los establecimientos de crédito: comparación crítica del estándar internacional y su implementación en Colombia. Colombia: Universidad Externado. Recuperado a partir de http://observatoriofinancieroybursatil.uexternado.edu.co/la-administracion-del-riesgo-de-credito-en-los-establecimientos-decredito-comparacion-critica-del-estandar-internacional-y-su-implementacion-en-colombia/

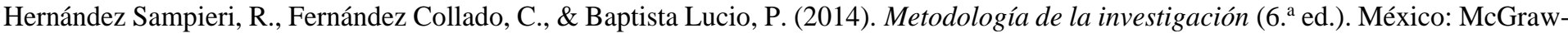
Hill Education.

Lara Rubio, J. (2010). La gestión del riesgo de crédito en las instituciones de microfinanzas (Tesis doctoral). Universidad de Granada. Recuperado a partir de digibug.ugr.es/bitstream/10481/5648/1/18892656.pdf

Macedo, G. (2014). Seminario Taller «Gestión del sistema financiero».

Maslach, C., \& Jackson, S. E. (1986). Maslach Burnout Inventory (3. ${ }^{\mathrm{a}}$ ed.). Palo Alto: Consulting Psychologists Press. Palo Alto, C. A. 19.

Mishkin, F. S. (2008). Moneda, banca y mercado financieros. México: Pearson.

Morales Guerra, M. L. (2007). La administraciòn del riesgo de crédito en la cartera de consumo de una institución bancaria (Tesis). Universidad de San Carlos de Guatemala, Guatemala. Recuperado a partir de http://www.biblioteca.usac.edu.gt/tesis/03/03_3045.pdf.

Murillo Torrecilla, A., \& Huamán Hurtado, B. (2012). Administración de Riesgo Crediticio y su Incidencia en la Morosidad de la Cooperativa de Ahorro y Crédito Chiquinquirá durante el periodo 2010, Huaraz (Tesis). Universidad Nacional Santiago Antúnez de Máyalo, Huaraz - Perú.

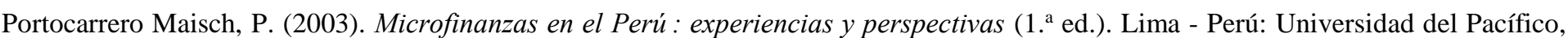
Centro de Investigación. Recuperado a partir de http://repositorio.up.edu.pe/handle/11354/141

Salvador, C. (2006). Gestión avanzada de riesgos de crédito: seminario para gerentes de riesgos de las entidades de microfinanzas Granada España. Lima - Perú: Superintendencia de Bancos y Seguro Banco Interamericano de Desarrollo. Recuperado a partir de http://www. sbs.gob.pe/basilea.pdf

Rev. Yachay volumen (7) Número (1), enero-diciembre 2018 




Stiglitz, J. E. (2007). El malestar en la globalización. México D.F: Punto de Lectura.

Suárez Ruiz, J., Varas Gómez, K., Sánchez Castro, P., Verástegui Valdera, I., \& Neyra Gonzales, M. (2013). Contribución de la gestión de los riesgos crediticios en su calificación de riesgo de la Caja Municipal de Ahorro y Crédito de Trujillo S. A. en el 2010 (Tesis). Universidad Privada Antenor Orrego. Recuperado a partir de http://es.slideshare.net/LyCanZz/trabajo-final-riesgos-la-gestion-delriesgo-crediticio-en-la-cmac-trujillo-grupo-07

Vela Meléndez, L., \& Uriol Chavez, J. (2012). Los factores que determinan la calidad de la cartera crediticia de las entidades microfinancieras de la Amazonía peruana en el periodo 2008 - 2011. Universidad Nacional Pedro Ruiz Gallo, Lambayeque, Perú. Recuperado a partir de http://docplayer.es/1643720-Los-factores-que-determinan-la-calidad-de-la-cartera.html
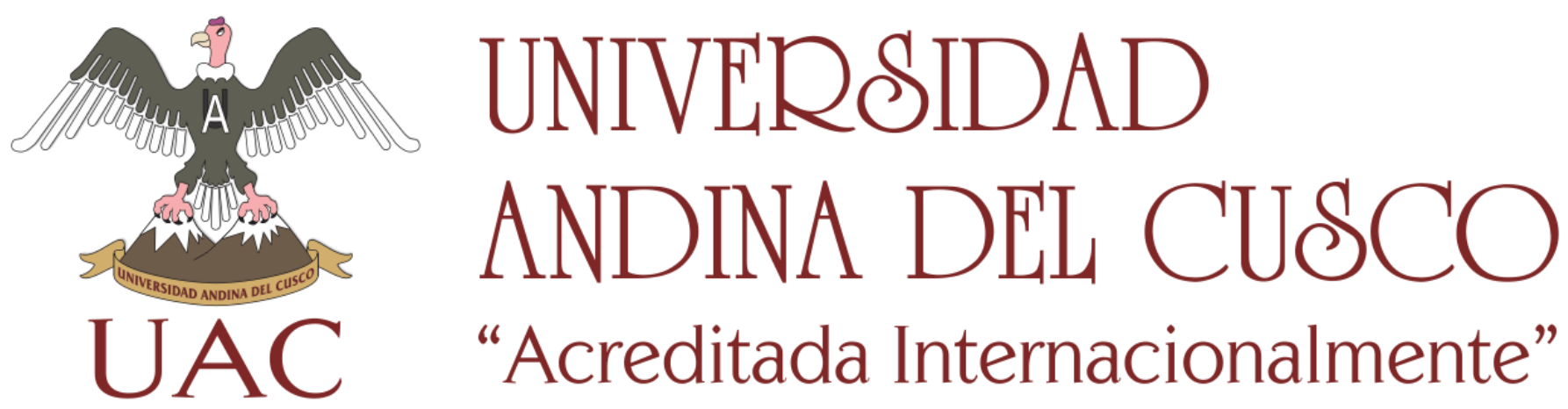